\title{
Effects of Lactobacillus rhamnosus GG as a probiotic on neonatal hyperbilirubinemia
}

\author{
Mehmet Mutluํ, Erdal Irmak ${ }^{2}$, Yakup Aslan¹, Şebnem Kader ${ }^{1}$ \\ ${ }^{1}$ Division of Neonatology, ${ }^{2}$ Department of Pediatrics, Karadeniz Technical University, Faculty of Medicine, Trabzon, Turkey. \\ E-mail: drmehmetmutlu38@hotmail.com \\ Received: 21st July 2017, Revised: 30th August 2017, Accepted: 3rd February 2018
}

SUMMARY: Mutlu M, Irmak E, Aslan Y, Kader Ş. Effects of Lactobacillus rhamnosus GG as a probiotic on neonatal hyperbilirubinemia. Turk J Pediatr 2018; 60: 482-487.

Undeveloped intestinal microflora and increased enterohepatic circulation of the newborn contribute to increase of plasma bilirubin level in the first days of life. This study was undertaken to determine the role of probiotic support started immediately after birth on neonatal hyperbilirubinemia in babies born by normal spontaneous vaginal delivery and breastfed only. A total of 150 healthy term newborns were included in the study and allocated in the study and control groups. Immediately after birth, newborns in the study group received probiotic in liquid drop form (Maflor ${ }^{\circledR}$ drops containing Lactobacillus Rhamnosus GG $10^{9}$ colony forming units, Mamsel Ilac, Turkey), at a dose of 5 drops a day, orally for 10 days. Newborns in the control group received 5 drops of saline solution per day orally, instead. Serum bilirubin levels (cord, $3^{\text {rd }}, 5^{\text {th }}$ and $10^{\text {th }}$ days of life) and defecation frequency were recorded. Mean total bilirubin levels on the $3^{\text {rd }}, 5^{\text {th }}$ and $10^{\text {th }}$ days were significantly lower and mean defecation frequency was significantly higher in the probiotic group compared to the controls $(\mathrm{p}<0.05$ for each). Negative correlations were determined between defecation frequency and the $3^{\text {rd }}, 5^{\text {th }}$ and $10^{\text {th }}$ day-total bilirubin levels $(\mathrm{r}=-0.804, \mathrm{p}<0.001 ; \mathrm{r}=-0.818$, $\mathrm{p}<0.001 ; \mathrm{r}=-0.648, \mathrm{p}<0.001$, respectively). Probiotic (Lactobacillus rhamnosus GG) support initiated immediately after birth has a positive effect on bilirubin metabolism and may reduce the risk of hyperbilirubinemia.

Key words: probiotic, hyperbilirubinemia, newborn, meconium.

Neonatal hyperbilirubinemia is a common problem in newborns. It is usually a benign and transient condition, but in some cases, persistent increase in bilirubin level may cause bilirubin induced neurological dysfunction and kernicterus. Main causes of hyperbilirubinemia in newborns are increased bilirubin load, insufficiency in bilirubin transport to the liver, hepatic intake and conjugation and increased enterohepatic circulation of bilirubin. ${ }^{1}$ The microflora of gastrointestinal tract plays an important role in regulation of increased bilirubin burden due to increased enterohepatic circulation in the newborn. The newborn's intestinal microflora is not fully formed in the first days of life and this causes increase in bilirubin enterohepatic circulation. ${ }^{2}$ It is reported that bilirubin return to the systemic circulation by the way of enterohepatic circulation increases serum bilirubin level by approximately $30 \% .^{3}$

In this study, we aimed to examine the effect of probiotic (Lactobacillus rhamnosus GG) support on serum bilirubin levels during the first 10 days in full-term normal vaginal spontaneous delivery newborns breastfed only.

\section{Material and Methods}

This prospective placebo-controlled study was conducted in the Department of Neonatology, Karadeniz Technical University Medical School between December 2014 and December 2015. The study protocol was approved by the Institutional Ethics Committee of Karadeniz Technical University (2014/115 24237859-120). Signed informed parental consent was obtained. 
A total of 150 term (gestational age $\geq 37-<42$ weeks), normal spontaneous vaginal delivery newborns with a birth weight between the $10^{\text {th }}-90^{\text {th }}$ percentiles, who had no need for intensive care and fed by breast milk only were enrolled in the study. Seventy-five newborns were allocated in the study or probiotic group (Group 1) and 75 in the control group (Group 2 ). The groups were formed by one of the case as study group and the other as control, respectively. In Group 1, the newborns received five drops of oral probiotic support [Maflor ${ }^{\circledR}$, Lactobacillus rhamnosus GG $10^{9}$ colony forming unit, Mamsel Pharmaceuticals, Turkey], while Group 2 had five drops of saline, once daily by the oral route, initiated immediately after birth and continued for ten days. Newborns who received probiotic were monitored for the potential of infection.

Newborns with familial hematologic disorders, signs of hemolysis due to blood group incompatibilities, bleeding into closed spaces due to birth trauma complications (e.g. cephalohematoma), suspected or documented neonatal infection such as chorioamnionitis, intrauterine infection, sepsis and urinary tract infection, perinatal and neonatal hypoxia, thyroid dysfunction, respiratory distress or insufficiency, metabolic and thermoregulatory dysfunction, hemodynamic instability and congenital heart disease, maternal phenobarbital usage history during the last month of the pregnancy, and venous hematocrit (Htc) levels $\geq 65 \%$ were excluded.

All mothers of the newborns were trained and encouraged about breastfeeding and benefits of breast milk in the prenatal and postnatal period. The basic approach to the potential problems that may be encountered in breastfeeding has been explained by our trained staff. During the control examination, nutritional status of the infants was questioned.
Gestational age, gender, birth weight, followup weights, first meconium evacuation time (in hours) and number of daily meconium evacuation of the newborns and maternal age were questioned and recorded. On followups information about the feeding pattern of the newborns was collected and further breastfeeding counseling was provided if needed.

Complete blood count, reticulocyte percentage, total and direct bilirubin levels, blood group, direct Coombs test and peripheral blood smear test results in cord blood samples and maternal blood group were recorded. On the $3^{\text {rd }}, 5^{\text {th }}$ and $10^{\text {th }}$ day-postnatal follow-ups, total and direct bilirubin levels, and on the $5^{\text {th }}$ day thyroid stimulating hormone and free thyroxin levels were measured. Cases with bilirubin levels over $12.9 \mathrm{mg} / \mathrm{dl}$ on the third days of life were noted. ${ }^{4}$ Samples for cord bilirubin levels, complete blood and reticulocyte counts, blood group analysis and direct Coombs tests and peripheral blood smear were obtained from cord. Total bilirubin levels were measured by direct spectrophotometry of the serum in a microhematocrit tube in the follow up.

\section{Statistical analyses}

All statistical analyses were performed using the Statistical Package for Social Sciences version 13.0 (SPSS Inc. Chicago, IL, USA). A priori sample size was calculated based on the assumptions of a type 1 error of $5 \%$ and type 2 error of $20 \%$ (power $80 \%$ ). Data were summarized as mean and standard deviation for metric, and count and \% for categorical variables. Comparison of categorical data was done by Chi square test. Distribution patterns of data were analyzed by one sample Kolmogorov-Smirnov test. Metric data, if normally distributed, were compared by unpaired Student t-test, and if non-normally

Table I. Demographic Features of the Newborns and Their Mothers.

\begin{tabular}{lccc}
\hline Demographics & $\begin{array}{c}\text { Group 1 } \\
(\mathrm{n}=75)\end{array}$ & $\begin{array}{c}\text { Group 2 } \\
(\mathrm{n}=75)\end{array}$ & $\mathrm{p}$ \\
\hline Gestational age, weeks & $39.0 \pm 1.3$ & $38.8 \pm 1.3$ & 0.460 \\
Birth weight, g & $3,254 \pm 346$ & $3,329 \pm 362$ & 0.199 \\
Male/female, n (\%) & $40(53.3) / 35(47.7)$ & $44(58.7) / 31(42.3)$ & 0.511 \\
Maternal age, years & $28.5 \pm 5.6$ & $29.3 \pm 5.3$ & 0.088 \\
& (range: $17-42)$ & Range $(18-41)$ & \\
\hline
\end{tabular}




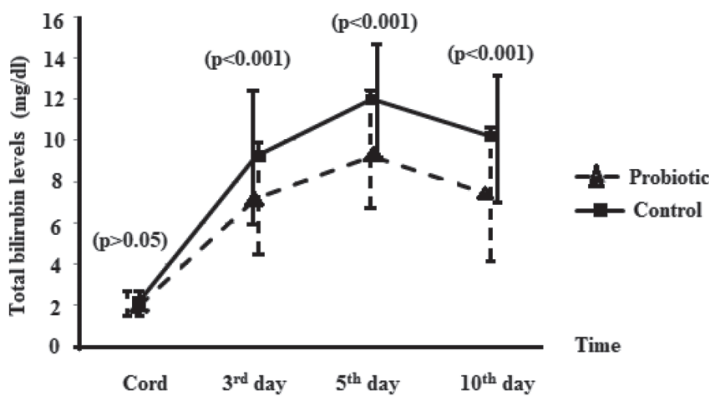

Fig 1. Serum total bilirubin levels during the study period.

distributed, with Mann-Whitney U test. Correlation of metric data was evaluated by bivariate Pearson correlation analysis. A p value $<0.05$ was defined as statistically significant.

\section{Results}

The newborns and their mothers included in the study were not significantly different between the study groups considering demographic characteristics $(p>0.05)$ (Table I). There was also no significant difference in body weight changes during follow-up between the two groups $(\mathrm{p}>0.05)$, and there was no pathologic body weight loss. Hemoglobin and hematocrit levels and reticulocyte counts of the study groups were not significantly different ( $p>0.05)$, (Table II).

No significant difference in mean total bilirubin levels in the cord blood of Groups 1 and 2 was detected $(p>0.05)$. On the other hand, probiotic group's $3^{\text {rd }}, 5^{\text {th }}$ and $10^{\text {th }}$ day samples had significantly lower bilirubin levels compared to controls $(\mathrm{p}<0.001)$ (Table II, Fig.1). Third, $5^{\text {th }}$ and $10^{\text {th }}$ day bilirubin levels of the Group 1 were found to be lower than Group 2 by $21.7,23.3$ and $27.4 \%$, respectively. None of the patients' bilirubin levels including that measured in the cord blood in both groups reached high levels requiring treatment such as phototherapy or exchange transfusion; however, bilirubin level exceeded $12.9 \mathrm{mg} / \mathrm{dl}$ in $14.6 \%$ of the probiotic supported newborns and $46.6 \%$ of the controls.

Mean time of the first meconium evacuation did not differ significantly among groups ( $p>0.05)$, but meconium evacuation in Group 1 on the $3^{\text {rd }}, 5^{\text {th }}$ and $10^{\text {th }}$ days was statistically more frequent than Group 2 ( $\mathrm{p}<0.001)$ (Table III).

Correlation analysis between stool frequency of the newborns and plasma bilirubin levels on the $3^{\text {rd }}, 5^{\text {th }}$ and $10^{\text {th }}$ days revealed significant negative correlations $(r=-0.804,<0.001$; $\mathrm{r}=-0.818, \mathrm{p}<0.001 ; \mathrm{r}=-0.648, \mathrm{p}=<0.001$, respectively). There was no infection in the newborns who received probiotic during the study or later. The situations that were not feasible immediately after birth such as sepsis, urinary tract infection, thyroid dysfunction, metabolic disorders etc. were not observed in the study group.

\section{Discussion}

This study was designed as a prospective controlled study to investigate the effects of probiotic support started immediately after birth on newborn jaundice in breastfed babies born by normal spontaneous vaginal delivery. This study is interesting for demonstrating the effect of a probiotic on lowering serum bilirubin levels through the first 10 days of life.

During early postnatal period, increased bilirubin load and insufficient metabolism confronts newborns to complications of increased bilirubin levels. The fetus, during its intrauterine life,

Table II. Laboratory Examinations of the Newborns.

\begin{tabular}{llll}
\hline Findings & $\begin{array}{l}\text { Group 1 } \\
(\mathrm{n}=75)\end{array}$ & $\begin{array}{l}\text { Group } 2 \\
(\mathrm{n}=75)\end{array}$ & $\mathrm{p}$ \\
\hline Hemoglobin, g/dl & $15.9 \pm 1.8$ & $16.5 \pm 2.0$ & 0.080 \\
Hematocrit, \% & $47.3 \pm 5.2$ & $48.6 \pm 5.5$ & 0.141 \\
Reticulocyte, \% & $2.4 \pm 0.8$ & $2.2 \pm 0.7$ & 0.131 \\
Cord bilirubin, mg/dl & $1.9 \pm 0.6(0.9-2.7)$ & $2.1 \pm 0.6(0.2-2.9)$ & 0.639 \\
$3^{\text {rd }}$ day bilirubin, mg/dl & $7.2 \pm 2.7(1.8-14.4)$ & $9.2 \pm 3.3(3.2-16.0)$ & $<0.001$ \\
$5^{\text {th }}$ day bilirubin, mg/dl & $9.2 \pm 3.3(1.6-16.1)$ & $12.0 \pm 3.1(5.2-18.1)$ & $<0.001$ \\
$10^{\text {th }}$ day bilirubin, mg/dl & $7.4 \pm 3.2(1.0-13.3)$ & $10.2 \pm 3.1(3.4-16.4)$ & $<0.001$ \\
\hline
\end{tabular}


is protected from the increased bilirubin load by the removal through the placenta. The role of $\beta$-glucuronidase activity has an important place in this placental bilirubin removal system. Persistence of increased $\beta$-glucuronidase activity after birth contributes to higher plasma bilirubin levels. To decrease this overload of bilirubin, meconium serving as a bilirubin storage site should be evacuated, $\beta$-glucuronidase enzyme activity should be suppressed, and bilirubin secreted into the intestine should be converted to urobilinoid immediately or disposed in the feces in its conjugated form. At this stage, intestinal flora has an important role, since it has been shown in experimental studies that intestinal flora and bilirubin metabolism has a close relationship. ${ }^{5-7}$

Intestinal flora is insufficient during the early neonatal period. Flora formation is affected by some external factors such as mode of delivery, maternal microbiota, feeding and use of antibiotics, and some intrinsic factors such as microbial factors and neonatal gastrointestinal structure. ${ }^{8}$ In our study, both groups consisted of healthy neonates were standardized in terms of several factors affecting gastrointestinal flora such as gestational age, route of delivery (normal spontaneous vaginal delivery), and feeding (breast feeding).

The newborn born via vaginal delivery is inoculated with a vaginal flora (mixture of gram-negative and gram-positive bacteria, aerobes and anaerobes). Bifidobacteria constitute the largest group within the infant intestinal flora. ${ }^{9}$ Lactobacilli, facultative anaerobic intestinal flora bacteria, mostly colonize in regions with high endogenous $\beta$-glucuronidase activity such as the stomach, duodenum and jejunum. ${ }^{10,11}$ Although lactobacilli were not dominant microbiota in the early days of newborns, these bacteria were preferred for this study due to there are studies that showed $\beta$-glucuronidase activity was suppressed by Lactobacillus rhamnosus GG. ${ }^{12,13}$ In our study, probiotic supplementation group has lower bilirubin levels. This situation may be related with suppressed $\beta$-glucuronidase activity, reduced enterohepatic circulation by preventing colonization of pathogens with high $\beta$-glucuronidase activity and acceleration of meconium passage.

Nutrition and frequency of defecation are important factors that may influence jaundice. In our study, mothers were informed about feeding and jaundice, and encouraged for breastfeeding. Because of this training program, we did not observe early breast milk jaundice due to insufficient breastfeeding and pathologic body weight loss. Parallel to our results, more than eight times a day breastfeeding during the first three days of life compared to less frequently fed neonates were reported to stimulate gastrocolic reflex which increased bowel motility and accelerated meconium passage that lowered bilirubin levels as a result. ${ }^{14}$

Delay in meconium passage causes increase in serum bilirubin levels and prolongs hyperbilirubinemia by enterohepatic circulation. ${ }^{15}$ In a study by Tribelli et al. ${ }^{3}$ enterohepatic circulation was found to contribute approximately $30 \%$ to the increase in total plasma bilirubin level. In line with the above report, we observed 21.7, 23.3 and $27.4 \%$ lower bilirubin levels in the probiotic supported newborns on the $3^{\text {rd }}, 5^{\text {th }}$ and $10^{\text {th }}$ days, respectively, compared to the controls. Our findings of statistically significant negative correlation between defecation frequency and serum bilirubin levels and increased defecation frequency among probiotic supported infants may be considered as another mode of action of probiotic-induced plasma bilirubin reduction.

Table III. Comparison of Defecation Characteristics of the Newborns

\begin{tabular}{llll}
\hline Defecation frequency & $\begin{array}{l}\text { Group 1 } \\
(\mathrm{n}=75)\end{array}$ & $\begin{array}{l}\text { Group 2 } \\
(\mathrm{n}=75)\end{array}$ & $\mathrm{P}$ \\
\hline First meconium evacuation time, hours & $4.0 \pm 2.9$ & $3.5 \pm 2.3$ & 0.419 \\
$3^{\text {rd }}$ day defecation frequency, n & $3.2 \pm 1.0$ & $2.5 \pm 0.9$ & $<0.001$ \\
$5^{\text {th }}$ day defecation frequency, n & $4.5 \pm 1.2$ & $2.9 \pm 0.9$ & $<0.001$ \\
$1^{\text {th }}$ day defecation frequency, n & $4.7 \pm 0.6$ & $3.6 \pm 0.6$ & $<0.001$ \\
\hline
\end{tabular}

Data is presented as mean \pm standard deviation 
There are a limited number of studies examining the effects of probiotics on neonatal jaundice and the results are conflicting. Demirel et al. ${ }^{16}$ reported that Saccharomyces boulardii supported infants with low birth weight and neonatal jaundice needed phototherapy for shorter periods of time most probably by decreasing feeding intolerance or suppressing bilirubin reuptake through enterohepatic circulation. On the other hand, Serce et al. ${ }^{17}$ reported Saccharomyces boulardii support did not lower bilirubin levels significantly in babies undergone phototherapy with gestational ages between 3542 weeks. Mu-Xue et al. ${ }^{18}$ determined jaundice in $33.3 \%$ of probiotic supported newborns and $57 \%$ in controls; and suggested that probiotics reduced neonatal jaundice frequency.

In our study, the threshold for plasma bilirubin level was accepted as $12.9 \mathrm{mg} / \mathrm{dl}$ on the third days of life as described by Bertini et al. ${ }^{4}$ since none of the newborns serum bilirubin levels reached the therapeutic limit described by American Academy of Pediatrics Subcommittee. ${ }^{19}$ We observed that $14.6 \%$ of the probiotic group and $46.6 \%$ of the control group had serum bilirubin levels higher than $12.9 \mathrm{mg} / \mathrm{dl}$.

The findings of our study are consistent with some of the data reported in the literature ${ }^{16,18}$, while contradicting another ${ }^{17}$ which is most probably due to the type and dose of probiotic used, and the time of administration (immediately after birth or when jaundice observed).

The limitations of this study are that bilirubin levels may be followed up with a transcutaneous bilirubin device to avoid blood taking procedures and none of the patients' bilirubin levels in both groups reached high levels requiring treatment. We do not have a transcutaneous bilirubin device. Bilirubin levels of the patients in both groups which did not reach high levels requiring treatment may make the supplementation of probiotic less meaningful.

In conclusion, we have observed statistically significant difference between the probiotic supported and control groups in terms of plasma bilirubin levels and defecation frequency on the $3^{\text {rd }}, 5^{\text {th }}$ and $10^{\text {th }}$ days in favor of the probiotic supplementation. Probiotic (Lactobacillus rhamnosus GG) support initiated immediately after birth has a positive effect on bilirubin metabolism and may be reduce the risk of hyperbilirubinemia. However, it was concluded that further studies with larger samples are needed on this topic.

\section{REFERENCES}

1. Kaplan M, Wong RJ, Sibley E, Stevenson DK. Neonatal jaundice and liver disease. In: Martin RJ, Fanaroff AA, Walsh MC (eds). Fanaroff and Martin's Neonatal Perinatal Medicine Diseases of the Fetus and Infant (9th ed) Vol. 2. Philadelphia: Elsevier Mosby, 2011: 1443-1496.

2. Khoury MJ, Calle EE, Joesoef RM. Recurrence risk of neonatal hyperbilirubinemia in siblings. Am J Dis Child 1988; 142: 1065-1069.

3. Tiribelli C, Ostrow JD. Intestinal flora and bilirubin. J Hepatol 2005; 42: 170-172.

4. Bertini G, Dani C, Tronchin M, Rubaltelli FF. Is breast feeding really favoring early neonatal jaundice? Pediatrics 2001; 107: E41.

5. Midtvedt T, Gustafsson BE. Microbial conversion of bilirubin to urobilins in vitro and in vivo. Acta Pathol Microbiol Scand B 1981; 89: 57-60.

6. Vitek L, Zelenka J, Zadinova M, Malina J. The impact of intestinal microflora on serum bilirubin levels. J Hepatol 2005; 42: 238-243.

7. Norin KE, Persson AK, Saxerholt H, Midtvedt T. Establishment of Lactobacillus and Bifidobacterium species in germfree mice and their influence on some microflora-associated characteristics. Appl Enviro Microbiol 1991; 57: 1850-1852.

8. Malmuthuge N, Griebel PJ, Guan le L. The gut microbiome and its potential role in the development and function of newborn calf gastrointestinal tract. Front Vet Sci 2015; 2: 36.

9. Houghteling PD, Walker WA. Why is initial bacterial colonization of the intestine important to the infant's and child's health? J Pediatr Gastroenterol Nutr 2015; 60: 294-307.

10. Guarner F, Malagelada JR. Gut flora in healthy hand disease. Lancet 2003; 361: 512-519.

11. Coşkun T. Probiyotikler, genel özellikleri ve etki mekanizmaları. Turkiye Klinikleri J Pediatr Sci 2012; 8: 1-11.

12. Ling WH, Korpela R, Mykkanen H, Salminen S, Hänninen O. Lactobacillus strain GG supplementation decreases colonic hydrolytic and reductive enzyme activities in healthy female adults. J Nutr 1994; 124 : 18-23.

13. Verma A, Shukla G. Probiotics Lactobacillus rhamnosus GG, Lactobacillus acidophilus suppresses DMH-induced procarcinogenic fecal enzymes and preneoplastic aberrant crypt foci in early colon carcinogenesis in Sprague Dawley rats. Nutr Cancer 2013; 65: 84-91.

14. De Carvalho M, Klaus MH, Merkatz RB. Frequency of breast-feeding and serum bilirubin concentration. Am J Dis Child 1982; 136: 737-738. 
15. Srinivasjois R, Sharma A, Shah P, Kava M. Effect of induction of meconium evacuation using per rectal laxatives on neonatal hyperbilirubinemia in term infants: A systematic review of randomized controlled trials. Indian J Med Sci 2011; 65: 278-285.

16. Demirel G, Celik IH, Erdeve O, Dilmen U. Impact of probiotics on the course of indirect hyperbilirubinemia and phototherapy duration in very low birth weight infants. J Matern Fetal Neonatal Med 2013; 26: 215218 .
17. Serce O, Gursoy T, Ovali F, Karatekin G. Effects of Saccharomyces boulardii on neonatal hyperbilirubinemia: A randomized controlled trial. Am J Perinatol 2015; 30: $137-142$.

18. Mu-Xue Y, Dong-Ping C, Zhong-Jiao Y, Yue-Xin L. The effect of probiotics on the incidence of neonatal hyperbilirubinemia. Chin J Microecology 2003; 5: 16.

19. American Academy of Pediatrics Subcommittee on Hyperbilirubinemia. Management of hyperbilirubinemia in the newborn infant 35 or more weeks of gestation. Pediatrics 2004; 114: 297-316. 